ИЗВЕСТИЯ АКАДЕМИИ НАУК ЭСТОНСКОИ ССР. ФИЗИКА * МАТЕМАТИКА

PROCEEDINGS OF THE ACADEMY OF SCIENCES OF THE ESTONIAN SSR. PHYSICS * MATHEMATICS

$1985,34,2$

\title{
ON THE LUMINOSITY, EFFECTIVE TEMPERATURE AND RADIUS OF P CYGNI
}

\author{
(Presented by G. Kuzmin)
}

For the further modelling of P Cygni's envelope and to specify our knowledge of P Cygni's physics and evolutionary stage, we must know the star's fundamental physical parameters. Below we shall discuss the luminosity, effective temperature and radius of the star, taking into account the observational data about the far ultraviolet, optical, infrared and radio fluxes.

1. Interstellar reddening, distance and absolute visual magnitude. Interstellar reddening, distance and absolute magnitude have previously been discussed by H. Van Schewick $\left[{ }^{1}\right]$. From the analysis of proper motions in the galactic cluster IC 4996 and the association Syg OBI it follows that P Cygni must be a member of the association. Therefore, we can use the mean association values $V-M_{v}=13.00, E(B-V)=0.62$, $A_{v}=1^{m} .86$ and $d=1700 \mathrm{pc}$ in the analysis of P Cygni characteristics.

These results are in qualitative accordance with $E(B-V)=0.56$. This value can be obtained from the analysis of the data on P Cygni's neighbouring stars $\left[{ }^{2}\right]$.

Our results may be challenged, however, taking into account the intensities of the interstellar absorption features. V. A. Ambartsumian et al. $\left[{ }^{3}\right]$ have used the values of the intensities of the wings in the interstellar $L_{\alpha}$ line and found that $E(B-V)=0.40 \pm_{0.10}^{0.20}$. They concluded that in the spectrum of $\mathrm{P}$ Cygni the intensities of interstellar bands at $\lambda 4430$ and $\lambda 5780$ lead to $E(B-V)=0.30$. They also affirmed that around P Cygni no stars with anomalously weak bands of interstellar absorption had been observed. Finally, they accepted $E(B-V)=$ $=0.35$. Estimating the probable distance between 0.6 and $1.8 \mathrm{kpc}$, they found that the absolute visual magnitude values are $-7 .^{m} 6>M_{v}>-5{ }^{m} 2$.

We shall discuss this problem in greater detail to find more plausible values of $E(B-V)$ etc.

Observational data on the far ultraviolet spectral region obtained from the satellites enable us to determine the colour-excess on the basis of the equivalent width of ultraviolet interstellar absorption band $\lambda 2175$.

K. Nandy et al. $\left[{ }^{4}\right]$ found that the equivalent width of $\lambda 2175$ band which is dependent on the colour-excess is reflected by the following relation

$$
W_{2175}=(450 \pm 30) E(B-V) .
$$

Using the ultraviolet spectrophotometric data obtained from the S2/68 experiment with the TD-1 satellite $\left[{ }^{5,6}\right]$, we have determined the colour-excesses $E(B-V)_{2175}$ for a score of normal hot stars with $E(B-V)$ known from photometriçal data, These values proved to be 


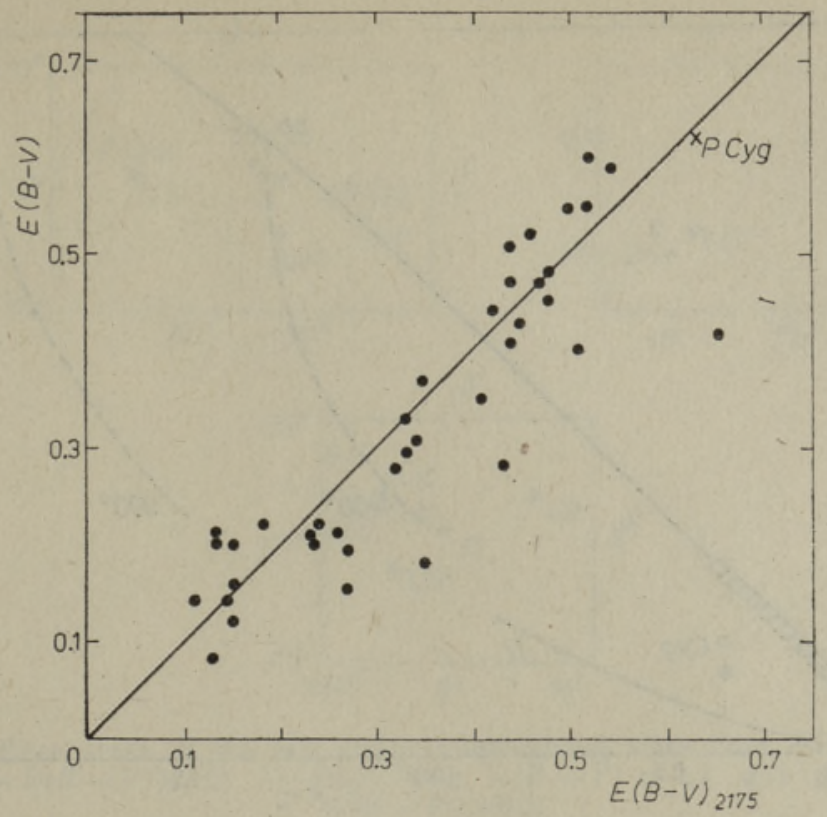

Fig. 1. The correlation between $E(B-V)_{2175}$ and photometric $E(B-V)$.

well correlated with each other (Fig. 1). Making use of the relation, we get $E(B-V)=0.63 \pm 0.08$ for P Cygni.

U. Hänni [ $\left.{ }^{7}\right]$ has developed a method for the determination of $E(B-V)$ from the given observational data of $\lambda 2175$ interstellar absorption band, using the gradual correction of the energy distributions from interstellar mutilations until the absorption in the band would disappear. He found $E(B-V)=0.62$ for P Cygni.

Thus, $E(B-V)_{2175}$ is in accordance with Van Schewick's photometrical determinations. We shall adopt the value $E(B-V)=0.62 \pm 0.05$.

The colour-excess may partly be caused by the circumstellar dust. V. A. Ambartsumian et al. ${ }^{[3]}$ have stated that the presence of the circumstellar dust is impossible in case of P Cygni as, according to the infrared observations carried out by $M$. J. Barlow and $M$. Cohen $\left[{ }^{8}\right]$, the whole infrared excess is caused by free-free radiation. The location of P Cygni on the $(H-K)$ versus $(K-L)$ diagram (Fig. 2) rules out all other mechanisms for infrared excess than that of free-free radiation. Thus, we affirm that the colour-excess is entirely interstellar. All these methods are based on the influence of the dust on the spectrum. Below we shall discuss anomalously weak interstellar bands in the spectrum of P Cygni.

L. M. Hobbs $\left[{ }^{9}\right]$ has published a well-known investigation of neutral natrium towards several distant stars. The correlation between the amounts of interstellar gas and dust is illustrated in Fig. 3. From Fig. 3 one can see that there exists a certain although ill-defined correlation between the amounts of gas and dust. The location of P Cygni in this plot shows that the interstellar matter in the direction of this star contains less interstellar gas than could be expected from the colour-excess.

We define

$$
\langle\lg N(\mathrm{NaI})-E(B-V)\rangle=\frac{\lg N(\mathrm{Na} \mathrm{I})-E(B-V)}{\lg N(\mathrm{Na} \mathrm{I})-E(B-V)}
$$

These values $\langle\lg N(\mathrm{Na} \mathrm{I})-E(B-V)\rangle$ enable us to discuss the ratio 


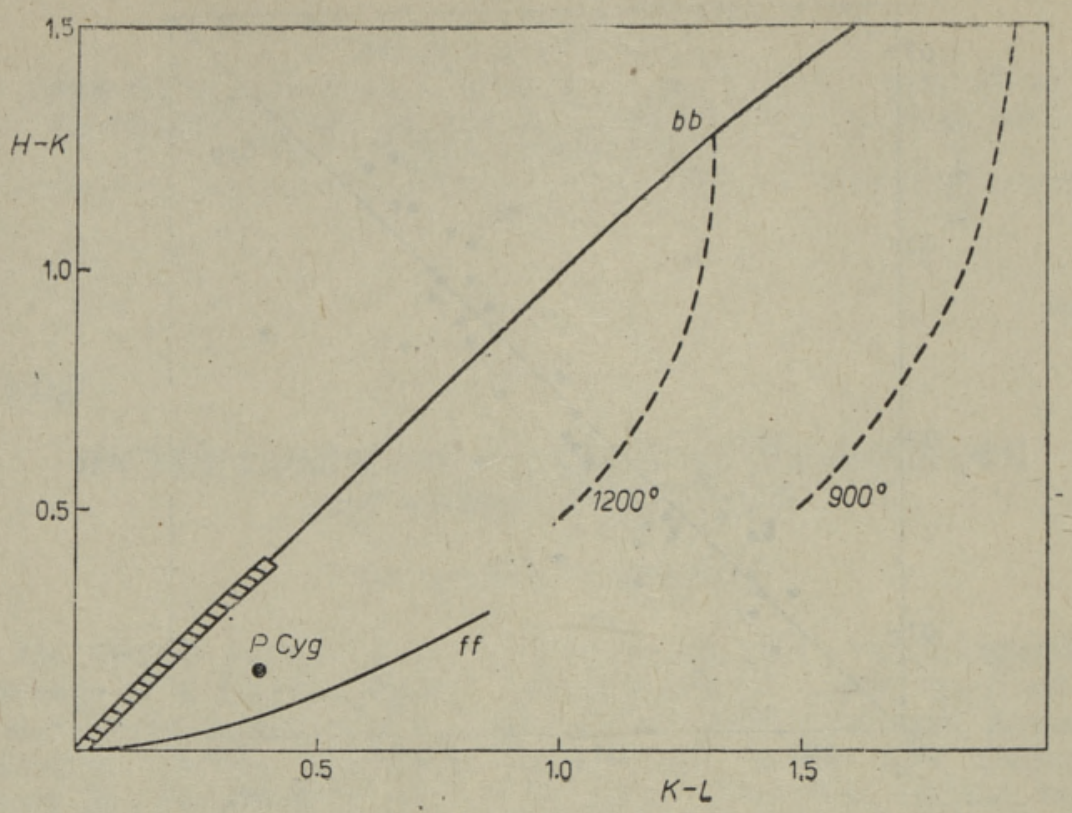

Fig. 2. The $(\mathrm{H}-\mathrm{K})$ versus $(\mathrm{K}-\mathrm{L})$ diagram. From the location of $\mathrm{P}$ Cygni it follows that free-free radiation must be the only source of the infrared excess of the star.

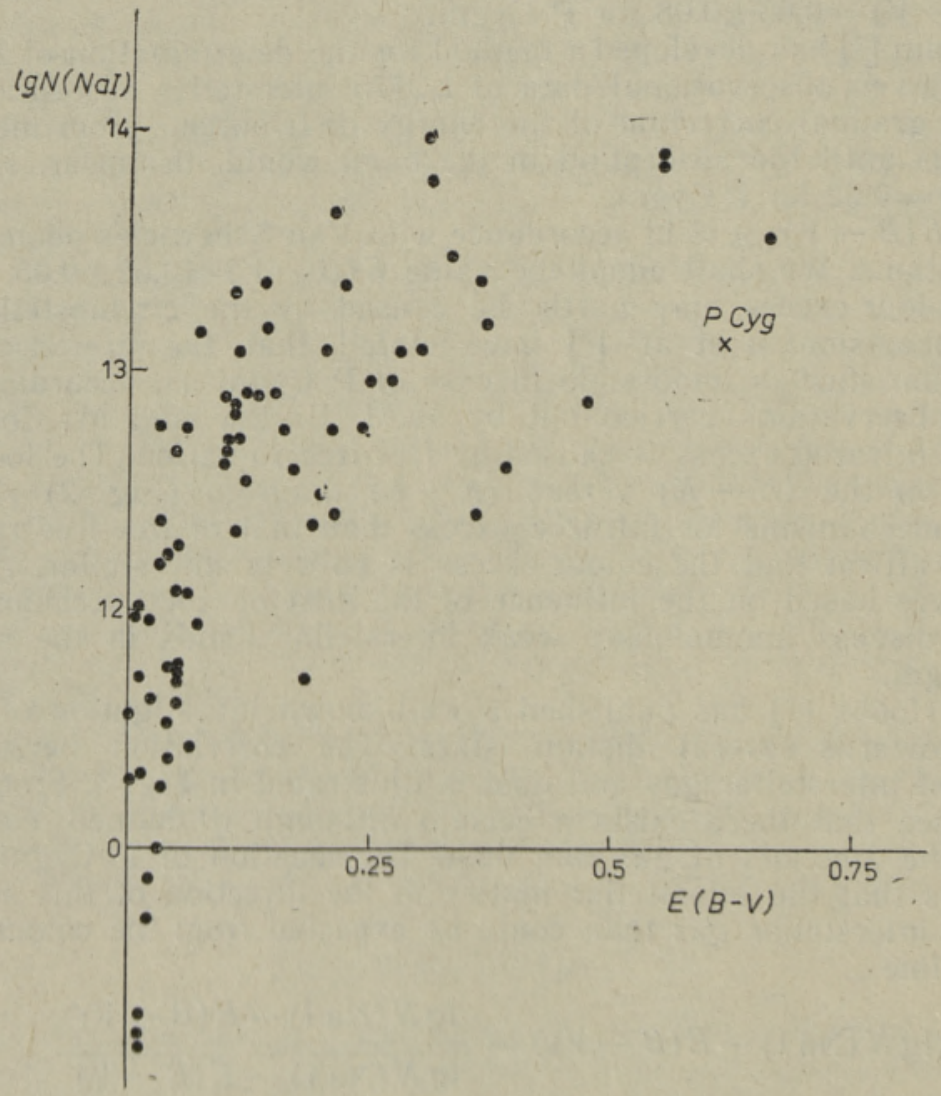

Fig. 3. The correlation between the amounts of $\mathrm{NaI}$ and $E(B-V)$. 

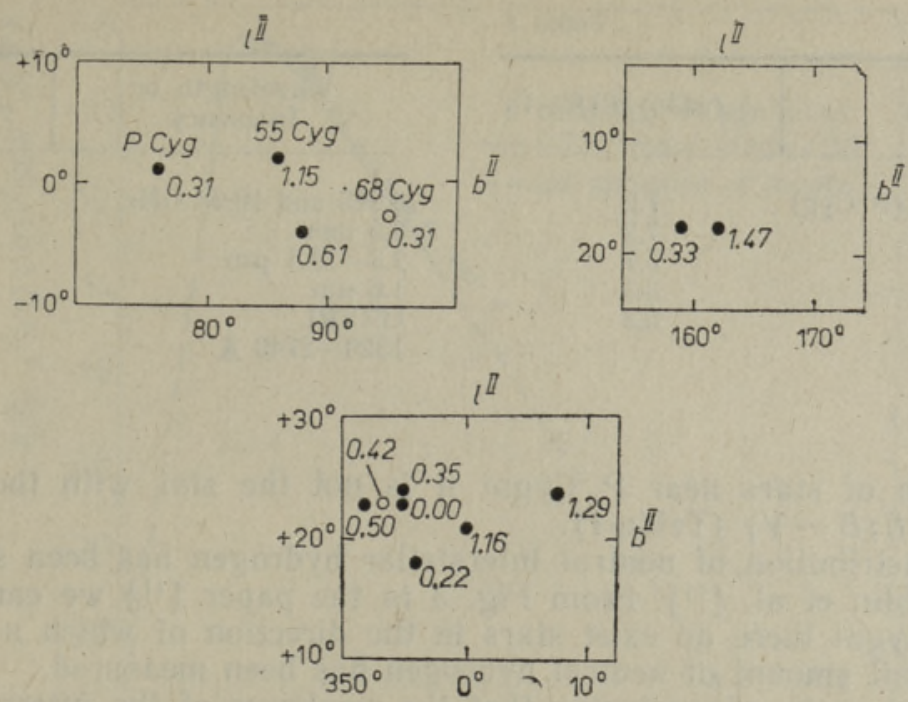

Fig. 4. The three areas in the sky where neighbouring stars have different values of $\langle\lg N(\mathrm{NaI})-E(B-V)\rangle$. $O-$ stars with $E(B-V) \leqslant 0.2$ and - stars with $E(B-V)>0.2$.

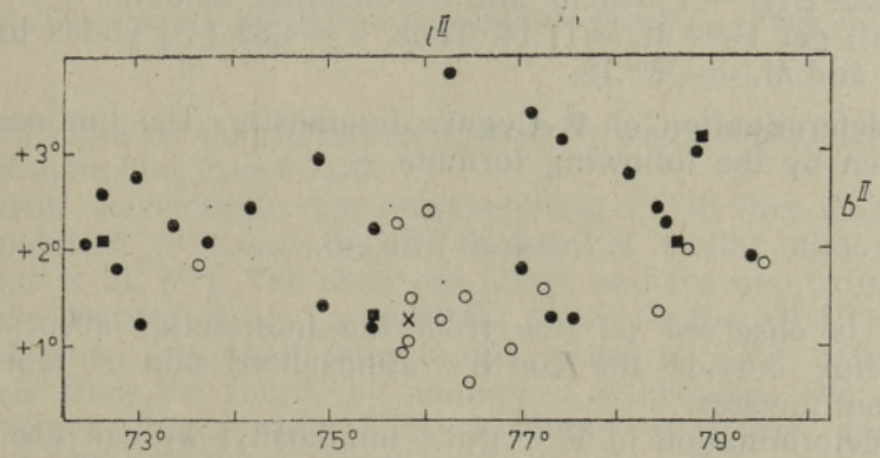

Fig. 5. The location of the stars with known $A_{c}(4430) / E(B-V)$ in the vicinity of $\mathrm{P}$ Cygni. $\mathrm{x}-\mathrm{P}$ Cygni, $\mathrm{O}-A_{\mathrm{c}}(4430) / E(B-V)<10,-10<A_{\mathrm{c}}(4430) / E(B-V)<15$ and $-A_{c}(4430) / E(B-V)>15$.

of gas and dust in the direction of individual stars within selected areas. In the direction of P Cygni the gas concentration makes up only one-third of the mean value. The stars around P Cygni with $E(B-V)<0.2$ have normal or nearly normal values. Such areas are not rare in the sky (Fig. 4). Therefore, the existence of stellar pairs with different ratios of interstellar gas and dust is rather a common phenomenon. In the direction of P Cygni an anomalously small amount of $\mathrm{Na} I$ has been observed if compared to the dust particles.

Below we shall discuss the interstellar absorption band intensities according to the catalogue presented by T. P. Snow et al. $\left[{ }^{10}\right]$. Using the values of the central intensities of the interstellar band $A_{c}(4430)$ and those of the colour-excesses, we can figure out the $A_{c}(4430) / E(B-V)$ characteristics. Fig. 5 represents the part of the sky in the surroundings of P Cygni where the stars are divided into groups according to the value of $A_{c}(4430) / E(B-V)$. From Fig. 5 it follows that near $P$ Cygni there exists an area with anomalously weak $\lambda 4430$ interstellar features, and a little further there is an area with strong interstellar $\lambda 4430$ bands. In 


\begin{tabular}{l|l}
\hline Star & $A_{c}(4430) / E(B-V)$ \\
\hline
\end{tabular}

HD 193237 (P Cyg

HD 193945

HD 193946

HD 194153

HD 197512

\section{2}

7.2

5.7

6.5

5.3

$\begin{gathered}\text { Wavelength or } \\ \text { frequency }\end{gathered}$
4.995 and $10.68 \mathrm{GHz}$
$3.3 \mathrm{~mm}$
$2.3-19.5 \mu \mathrm{m}$
$1.6 \mu \mathrm{m}$
UBVRI
$1320-2740 \AA$

Source

4.995 and $10.68 \mathrm{GHz}$

$3.3 \mathrm{~mm}$

$2.3-19.5 \mu \mathrm{m}$

$1.6 \mu \mathrm{m}$

$1320-2740 \AA$ $\left[\begin{array}{l}13 \\ {[14}\end{array}\right]$
$[15]$
$[16]$
$[12]$
$[6]$

the group of stars near P Cygni it is not the star with the smallest $A_{c}(4430) / E(B-V)$ (Table 1$)$.

The distribution of neutral interstellar hydrogen has been studied by R. C. Bohlin et al. $\left[{ }^{11}\right]$. From Fig. 3 in the paper $\left[{ }^{11}\right]$ we can see that near P Cygni there do exist stars in the direction of which a comparatively small amount of neutral hydrogen has been measured.

We can make a conclusion that the weakness of the interstellar features which shows the influence of the interstellar gas in the direction of P Cygni must rather be taken for a not very strong anomaly of interstellar matter composition than a sign of proximity.

If we take $E(B-V)=0.62$ and the distance modulus $V-M_{v}=13.00$ $\left[{ }^{1}\right]$, we shall get $V_{0}-M_{v}=11.14$. Thus, $V=4.82\left[{ }^{12}\right]$ yields to $V_{0}=2.96$, $d=1700 \mathrm{pc}$ and $M_{v}=-8^{m} .18$.

2. Direct determination of $\mathbf{P}$ Cygni's luminosity. The luminosity of the star is given by the following formula -

$$
L=4 \pi d^{2} \int_{0}^{\infty} F_{E, \lambda} d \lambda,
$$

where $F_{E, \lambda}$ is observed as free from the interstellar absorption monochromatic flux outside the Earth's atmosphere and $d$ represents the distance from the star.

In the determination of P Cygni's luminosity, we can use the observational data on the fluxes from the bibliographical sources listed in Table 2 .

Interstellar reddening data have been corrected on the basis of the wavelength dependencies according to L. Luud $\left[{ }^{17}\right]$ and A. Sapar and I. Kuusik $\left[{ }^{18}\right]$.

We have observed the fluxes on a very large range of wavelengths, therefore the whole spectrum which gives a remarkable contribution to the luminosity is known. Therefore, we shall describe the calculation of the integral in parts. We have

$$
\begin{aligned}
\frac{L}{4 \pi d^{2}} & =\int_{0}^{\infty} F_{E, \lambda} d \lambda=\int_{0}^{166 \AA} F_{E, \lambda} d \lambda+\int_{166 \AA}^{515 \AA} F_{E, \lambda} d \lambda+\int_{515 \AA}^{912 \AA} F_{E, \lambda} d \lambda+ \\
& +\int_{912 \AA}^{1320 \AA} F_{E, \lambda} d \lambda+\int_{1320 \AA}^{3.3 \mathrm{~mm}} F_{E, \lambda} d \lambda+\int_{3.3 \mathrm{~mm}}^{4.995 \mathrm{GHz}} F_{E, \lambda} d \lambda+\int_{4.995 \mathrm{GHz}}^{\infty} F_{E, \lambda} d \lambda= \\
& =\Omega_{1}+\Omega_{2}+\Omega_{3}+\Omega_{4}+\Omega_{5}+\Omega_{6}+\Omega_{7} .
\end{aligned}
$$

Some of these integrals have been determined from the observed fluxes: $\Omega_{5}=7.89 \cdot 10^{-0}$. The value of the next integral $\Omega_{6}$ (also determined from the 


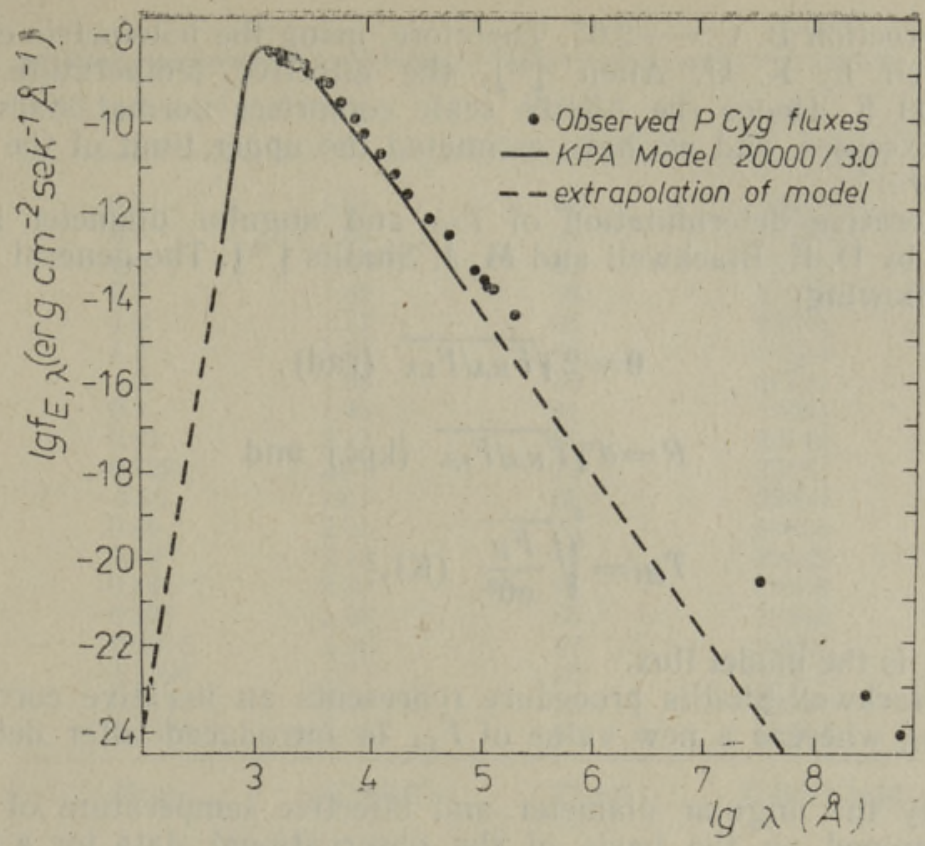

Fig. 6. The observed and interpolated absolute fluxes $F_{E, \lambda}$ from P Cygni.

observational data on the fluxes) is $2.60 \cdot 10^{-13}$, which makes only $3 \cdot 10^{-3} \%$ of $\Omega_{5}$. We assume that $\Omega_{6}=\Omega_{7}=0$.

For shorter wavelengths the extrapolation of the flux must be used. Our extrapolation is based on the models of stellar atmospheres by R. L. Kurucz et al. $\left.{ }^{19}\right]$. The observed fluxes and the approximation with the model by Kurucz et al. $T_{\text {eff }}=20000 \mathrm{~K}$ and $\lg g=3.0\left[{ }^{19}\right]$ are given in Fig. 6. Further, we shall refer to the model as KPA 20000/3.0, and other models from the source by analogous symbolism. The accordance of the observed fluxes with KPA $20000 / 3.0$ is rather good at the wavelengths $1320-10000 \AA$. Further in the infrared region an excess of the free-free radiation has been observed. Taking into consideration that the relative flux distribution is only slightly dependent on the KPA models of near temperatures, we shall use the fluxes given by KPA $20000 / 3.0$ for extrapolated ultraviolet fluxes.

In our integrals the bounders have been chosen under the following circumstances: $912 \AA$ is the Lyman continuum limit (physical consideration), $515 \AA$ is the limiting wavelength for the calculations in KPA $20000 / 3.0$ (mathematical consideration) and $166 \AA$ is the equal flux point with the last infrared point included in the calculation of the integral $\Omega_{7}$.

We have the following integrals $-\Omega_{4^{\prime}}=2.56 \cdot 10^{-6}, \Omega_{3}=4.96 \cdot 10^{-7}$ and $\Omega_{2}=3.16 \cdot 10^{-11} \cdot \Omega_{3}+\Omega_{4}+\Omega_{5}=1.095 \cdot 10^{-5}$. The value of integral $\mathfrak{\Omega}_{2}$ is only $3 \cdot 10^{-6} \%$ of the sum $\Omega_{3}+\Omega_{4}+\Omega_{5}$, and therefore we shall assume that $\Omega_{1}=$ $=\Omega_{2}=0$. Our integral is found within the accuracy limits of observations that are less than $5 \%$ for bright objects like P Cygni.

Thus, we shall have $L=3.79 \cdot 10^{39} \mathrm{erg} / \mathrm{s}, \lg L / L_{\odot}=6.00 \pm 0.05$ and $M_{\mathrm{Bol}}=-10^{m} \cdot 25$.

3. The effective temperature and radius of $\mathbf{P}$ Cygni which follow from the luminosity, distance, observed and model fluxes. We shall start from the zero approximation temperature which may be obtained from the $M_{\mathrm{Bol}}=-10^{m} .25$ and $M_{v}=-8^{m} .18$. These magnitudes give for the bolo- 
metric correction B. C. $=-2.07$. Therefore, using the bolometric correction scale given by K. U. Allen $\left[{ }^{20}\right]$, the effective temperature will be $T_{\text {efr }} \leqslant 22400 \mathrm{~K}$. Hence the Allen's scale comprises normal stars without infrared excesses, and we have estimated the upper limit of the effective temperature.

The iterative determination of $T_{\text {eff }}$ and angular diameter has been described by D. E. Blackwell and $M$. J. Shallis $\left[{ }^{21}\right]$. The general formulae are the following:

$$
\begin{gathered}
\theta=2 \sqrt{F_{E, \lambda} / F_{s, \lambda}} \quad(\mathrm{rad}), \\
R=d \sqrt{F_{E, \lambda} / F_{s, \lambda}} \quad(\mathrm{kpc}) \text { and } \\
T_{\text {eff }}=\sqrt[4]{\frac{F_{E}}{\sigma \theta^{2}}} \quad(\mathrm{~K}),
\end{gathered}
$$

where $F_{s, \lambda}$ is the model flux.

The Blackwell-Shallis procedure represents an iterative correction of $\theta$ and $T_{\text {eff }}$, whereas a new value of $F_{s, \lambda}$ is introduced after determining $T_{\text {eff. }}$

Usually the anguiar diameter and effective temperature of the star are determined on the basis of the observational data for a moderate quantity of wavelengths, mostly for the infrared region where the dependence on the atmospheric model for the determination of the quantities $F_{s, \lambda}$ is quite weak. In case of $\mathrm{P}$ Cygni, we have a remarkable infrared excess caused by free-free transitions in the star's outer envelope. According to T. Nugis et al. $\left[{ }^{32}\right]$ the effective radius in the infrared and radio wavelengths is significantly greater than the star's photosphere at ultraviolet wavelengths. For $\lambda=8.7 \mu \mathrm{m}$ the effective radius exceeds the photospheric radius $\sim 1.75$ times. Therefore, the extrapolated flux from the adjusted KPA 20000/3.0 model has been used for the photospheric infrared flux $F_{E, \lambda}$.

Here, two main aspects need further discussion. The calculations with the corrected data on the observed infrared fluxes received by model approximation introduce the dependence of $F_{E, \lambda}$ on the same model that has been used for the determination of $F_{s, \lambda}$. Therefore, the values of $T_{\text {eff }}$ and $\theta$ with the fluxes on the wavelengths greater than $1 \mu \mathrm{m}$, must be incorrect. The somewhat higher radii and lower temperatures in case of the wavelengths $2770-10000 \AA$ are probably caused by observational errors at shorter wavelengths and the non-considered free-free excess at longer wavelengths. The agreement of the values of $T_{\text {eff }}$ and $\theta$ found from far ultraviolet and other fluxes, shows that they really reflect the photosphere of the star. The discrepancies between the determined values are higher than could be expected from the observational and model errors (Table 3 ). The latter does not exceed $\sim 10 \%$ in $F_{E, \lambda} / F_{s, \lambda}$, $\sim 4 \%$ in the radius and $\sim 2 \%$ in the temperature. Therefore the calculation of the next approximation seems to be reasonable.

The confinement with the first approximation is justified by having quite similar results in all the discussed wavelengths and difficulties in finding $F_{s, \lambda}$ in the following approximation. It must be noted that the correction of the effective temperature by some hundreds of degrees with the scatter of single values about a thousand degrees is not required in case the effective temperature is about $10^{4}$ degrees.

We can check the inner correctness of our determinations, using the following expression -

$$
\lg L / L_{\odot}=4 \lg T_{\text {eff }}=2 \lg R / R_{\odot}-15.045,
$$




\begin{tabular}{l|c|c|c}
\hline$\lambda, \mu \mathrm{m}$ & $\theta \cdot 10^{4} \mathrm{rad}$ & $R / R_{\odot}$ & $T_{\text {eft } \mathrm{K}}$ \\
\hline & & & \\
6.5 & 1.81 & 68 & 22020 \\
5.0 & 1.86 & 69 & 21762 \\
4.0 & 1.85 & 69 & 21782 \\
2.7 & 1.82 & 68 & 21966 \\
1.8 & 1.77 & 66 & 22306 \\
1.2 & 1.87 & 70 & 21679 \\
1.0 & 1.86 & 70 & 21731 \\
0.9 & 1.42 & 91 & 19024 \\
0.83 & 2.17 & 81 & 20140 \\
0.725 & 2.01 & 75 & 20907 \\
0.56 & 1.81 & 68 & 22034 \\
0.46 & 2.01 & 75 & 20918 \\
0.40 & 2.18 & 82 & 20082 \\
0.36 & 2.30 & 86 & 19563 \\
0.274 & 2.28 & 85 & 19648 \\
0.2015 & 1.80 & 67 & 22090 \\
0.1598 & 1.74 & 65 & 22478 \\
0.1422 & 1.67 & 62 & 22970 \\
0.1370 & 1.86 & 69 & 21762 \\
\hline Mean & $1.95 \pm 0.21$ & $73 \pm 8$ & $21400 \pm 1100$
\end{tabular}

which assumes that $T_{\text {eff }}(\odot)$ is $5770 \mathrm{~K}$. If we calculate using the mean $T_{\text {eff }}$ and $R$, we shall get 6.00 . This value exactly coincides with the determined value in part 2 .

We conclude that $\theta=(1.95 \pm 0.21) \cdot 10^{-9} \mathrm{rad}=(4.02 \pm 0.44) \cdot 10^{-4}$ arc sec, $R=73 \pm 8 \mathrm{R}_{\odot}$ and $T_{\mathrm{eff}}=214 \overline{00} \pm 1100 \mathrm{~K}$.

4. Concluding remarks. Our results are in partial disagreement with those obtained by A. B. Underhill $\left[{ }^{23}\right]$. She applied the underestimated value for $E(B-V)$ by V. A. Ambartsumian et al. $\left[{ }^{3}\right]$ Therefore, she had energy distributions not completely corrected form the interstellar reddening. The comparison of energy distributions found with the colour-excesses 0.35 and 0.61 (Fig. 2 in $\left[{ }^{23}\right]$ ) with KPA models gives, in our opinion, a much better accordance in case of $E(B-V)=0.61$. Probably the arbitrary preferring of the lower value is a result of «esprit de contradiction» which would certainly adorn scientific discussions. The angular diameter of P Cygni is in good agreement due to the methods used. Therefore, the only principal source of discrepancies is the adoption of different colour-excesses. In the first part of our paper we have shown that the higher value of interstellar absorption should be preferred.

Another aspect to be discussed is the disagreement of the distance $1.7 \mathrm{kpc}$ adopted by the author with $1.2 \mathrm{kpc}$ given by C. S. Beals $\left[{ }^{24}\right]$. This value is the mean of the values determined by different methods and we shall discuss them separately. The analysis of the distance of NCC $687 \mathrm{i}$ presented by C.S. Beals according to $\mathrm{H}$. Trumpler [ $\left.{ }^{25}\right]$ presents an underestimated value. The adopted interstellar absorption $0^{m} .7$ per $\mathrm{kpc}$ is approximately 2.5 times smaller than the true value. The distance determined from the equivalent widths of the interstellar lines has also been underestimated because in the direction of $\mathrm{P}$ Cygni a relatively low gas abundance in interstellar matter has been observed. The only method which needs no corrections is the determination of the distance on the basis of the relationship between the distance and differential velocity due to the galactic rotation. The value of the distance $1.6 \mathrm{kpc}$ estimated by this method is very close to the value adopted by the author. Thus, the only correct value suggests that our estimation of the distance must be valid. 
Taking into account that our determinations are in good agreement with those presented by other investigators, we shall adopt the following complex of P Cygni's parameters:

$$
\begin{aligned}
E(B-V) & =0.62 \\
d & =1.7 \mathrm{kpc} \\
M_{v} & =-8^{m} .18 \\
L & =3.79 \cdot 10^{39} \mathrm{erg} / \mathrm{s} \\
\lg L / L_{\odot} & =6.00 \pm 0.05 \\
M_{\text {Bol }} & =-10^{m} .25 \\
\text { B.C. } & =-2.07 \\
T_{\text {eff }} & \leqslant 22400 \mathrm{~K} \quad \text { (from B.C.) } \\
\theta & =4.02 \pm 0.44 \text { marcs } \\
R & =73 \pm 8 \mathrm{R}_{\odot} \\
T_{\text {eff }} & =21400 \pm 1100 \mathrm{~K} \text { (from } L \text { and } \theta \text { ). }
\end{aligned}
$$

The author is very grateful to Prof. $\mathrm{H}$. Lamers for the preprint of the paper $\left[{ }^{26}\right]$ which inspired the author to wind up the investigations of P Cygni's fundamental parameters. Thanks are also due to Mrs. K. Ummik and Miss E. Turgulainen for their kind assistance.

\section{REFERENCES}

1. Van Schewick. H. Z. f. Astrophysik, 68, 229-234 (1968).

2. Лууд Л. Публ. Тартуск. астрофиз. обс., 35, 189-196 (1966).

3. Ambartsumian, V. A., Mirzojan, L. V., Snow, T. P. Астрофизика, 14, 425-438 (1978).

4. Nandy, K., Thompson, I. G., Jamar, C., Monfils, A., Wilson, R. Astron. Astrophys., 44, 195-207 (1976).

5. Jamar, C., Macau-Hercot, D., Monfils, A., Thompson, G. I., Houziaux, L., Wilson, R. Uitraviolet Bright-Star Spectrophotometric Catalogue. Paris, ESA, 1976.

6. Macau-Hercot, D., Jamar, C., Monfils, A., Thompson, G. I., Houziaux, L., Wilson, R. Supplement to the Ultraviolet Bright-Star. Spectrophotometric Catalogue. Paris, ESA, 1978 .

7. Hänni, U. ENSV TA preprint A-4. Tartu, 1981.

8. Barlow, M. J., Cohen, M. Astrophys. J., 214, 731-735 (19i7).

9. Hobbs, L. M. Astrophys. J. Suppl. Ser., 38, 129-146 (1978).

10. Snow, T. P., York, D. G., Welty, D. E. Astron. J., 82, 113-128 (1977).

11. Bohlin, R. C., Savage, B. D., Drake, J. F. Astrophys. J., 224, 132-142 (1978).

12. Johnson H. L., Mitchell, R. I., Iriarte, B., Wishniewski; W. Z. Comm. Lunar and Planetary Laboratory, 4, 99-110 (1966).

13. Wendker, H. J., Baars, J. W. M., Altenhoff, W. J. Nature Phys. Sci., 245, 111-119 (1973).

14. Schwartz, P. R., Spender, J. H. Mon. Notic. Roy Astron. Soc., 180, 297-303 (1977).

15. Gehrz, R. D., Hackwell, J. A., Jones, T. W. Astrophys. J., 191, 675-684 (1974).

16. Allen, D. Mon. Notic. Roy Astron. Soc., 161, 145-166 (1973).

17. Лууд Л. Публ. Тартуск. астрофиз. обс., 46, 45-54 (1978).

18. Sapar, A., Kuusik, I. Tartu Obs. Publ., 46, 71-84 (1978).

19. Kurucz, R. L., Peytremann, E., Avrett, E. H. Blanketed Model Atmospheres for Early-Type Star. Smithonian Institution, Washington D. C., 1974.

20. Аллен К. У. Астрофизические величины. М., «Мир», 1977.

21. Blackwell, D. E., Shallis, M. J. Mon. Notic. Roy. Astron. Soc., 180, 177-192 (1977).

22. Нугис Т., Колка Н., Лууд Л. Публ. Тартуск. астрофиз. обс., 47, 191-221 (1979).

23. Underhill, A, B. Astrophys. J., 234, 528-537 (1979).

24. Beals, C. S. Publ. Dominion Astrophys. Obs., 9, 1-137 (1951).

25. Trumpler, $H$. Lick Obs. Bull., 14, $154-167$ (1930).

26. Lamers H. J. G. L. M., de Groot, M., Cassatella, A. Astron. Astrophys, 123, L8-L10 (1983). 


\section{LUUD}

\section{P CYGNI KIIRGUSVOIMEST, EFEKTIIVSEST TEMPERATUURIST JA RAADIUSEST}

On uuritud interstellaarset neeldumist P Cygni suunas ja, rakendades saadud parandeid absoluutühikutes antud kiirgusvoole, leitud tähe põhilised füüsikalised karakteristikud. Kiirgusvõime $L=3,79 \cdot 10^{39} \mathrm{erg} / \mathrm{s}, \lg L / L_{\odot}=6,00 \pm 0,05$, raadius $R=73 \pm 8 \mathrm{R}_{\odot}$ ja efektiivne temperatuur $T_{\text {eft }}=21400 \mathrm{~K}$.

\section{л. лууд}

\section{О СВЕТИМОСТИ, ЭФФЕКТИВНОИ ТЕМПЕРАТУРЕ И РАДИУСЕ Р ЛЕБЕДЯ}

Исследовано межзвездное поглощение в направлении Р Лебедя. Полученные поправки применены для исправления наблюдаемых в абсолютных единицах потоков с последующим определением основных физических параметров звезды. Установлено, что светимость Р Лебедя $L=3,79 \cdot 10^{39}$ эрг $/ \mathrm{c}, \lg L / L_{\odot}=6,00 \pm 0,05$, радиус $R=73 \pm 8 \mathrm{R}_{\odot}$ и эффективная температура $T_{\text {eft }}=21400 \mathrm{~K}$. 